\title{
NON METRIZABILITY OF SOME TOPOLOGIZABLE ALGEBRAS
}

\author{
Zine El Abidine AbDelali
}

\begin{abstract}
We compare topologization of certain algebras as topological algebras as well as metrizable semitopological algebras. The main aim of the paper is to prove that the algebra of continuous functions on a non Lindelöf paracompact space is not topologizable as a metrizable semitopological algebra. This gives us further examples of locally convex topologizable algebras that are not topologizable as metrizable semitopological algebras. On the other hand we prove that the free algebra generated by $\boldsymbol{\aleph}$ variables has at last $\max \left\{2^{\boldsymbol{\aleph}_{0}}, \boldsymbol{\aleph}\right\}$ topologies of metrizable semitopological locally convex algebras. We provide also, for all $p \in(0,1]$, an example of locally bounded topological algebra which is not topologizable as a metrizable semitopological locally $p$-convex algebra. This is an investigation in the direction of some results of J. Esterle, V. Müller, W. Żelazko and the author in previous papers.
\end{abstract}

Mathematics subject classification (2010): 46H05. ordinals.

Keywords and phrases: Topological spaces, topological algebras, metrizable semitopological algebras,

\section{REFERENCES}

[1] Z. Abdelali, Topologisation d'une algèbre radicale non métrisable, Bulletin Greek Mathematical Society, (accepted paper [Ref.:EME-282, 14 March 2002]).

[2] Z. Abdelali, M. Chidami, Exemples séparant certaines classes d'algèbres topologiques, Proc. Amer. Math. Soc., 129, 6 (2000), 1763-1767.

[3] Z. Abdelali, M. ChidAmi, Non unicité des topologies localement-convexes complètes sur certaines algèbres, Commentationes Math., XLII, 2 (2002), 125-136.

[4] Z. Abdelali, M. Chidami, Topologisation et multiplication dans certaines algèbres, Contemporary Math. 427 (2007), 1-5.

[5] N. Bourbaki, Théorie des ensembles, Chapitre 3, Fascicule XX.

[6] N. Bourbaki, Topologie générale, Chap. 1 à 4, Masson, Paris, 1990.

[7] N. Bourbaki, Topologie générale, Chap. 5 à 10, Hermann, Paris, 1974.

[8] J. Esterle, Sur la métrisabilité de certaines algèbres d'opérateurs, Rev. Roumaine Math. Pures Appl., 24 (1979), 1157-1164.

[9] R. Frankiewicz, D. Plebanek, An example of a non-topologizable algebra, Studia. Math., 116 (1995), 85-87.

[10] L. Gillman, M. JeRISOn, Rings of continuous functions, Springer-Verlag, New York Heidelberg Berlin, 43, 1976.

[11] J.L. KelLey, General topology, Springer-Verlag, New York Heidelberg Berlin, 27, 1975.

[12] A. KоKK, W. ŻELAZKO, On vector spaces and algebras with maximal locally pseudoconvex topologies, Studia Math., 112 (1995), 195-201.

[13] G. Kӧтне, Topological vector spaces I, Springer-Verlag, Berlin Heidelberg New York, 159, 1983.

[14] A. Mallios, Topological algebras, Selected topics, North, Holland Math Studies 124, Amsterdam, 1986.

[15] E.A. MichaEL, Locally multiplicatively convex topological algebras, Mem. Amer. Math. Soc., 11 (1952).

[16] V. MülLER, On topologizable algebras, Studia. Math., 99, 2 (1991), 149-153.

[17] J.R. Munkres, Topology a first course, Prentice-Hall, Inc., Englewood Cliffs, New Jersey, 1975. 
[18] L. Waelbroeck, Topological vector spaces and algebras, Lecture Notes in Math, 230, Springer, 1991.

[19] M. WoJCIECHOWS KI, W. ŻELAZKo, Non-uniqueness of topology for algebras of polynomials, Colloq. Math., 71 (1997), 111-121.

[20] W. ŻELAZKo, A non-Banach m-convex algebra all of whose closed commutative subalgebras are Banach algebras, Studia. Math., 119, 2 (1996), 195-198.

[21] W. ŻELAZKo, A non-locally convex topological algebra with all commutative subalgebras locally convex, Studia. Math., 120, 1 (1996), 89-94.

[22] W. ŻELAZKo, Concerning topologization of algebras-the results and open problems, in: Advances in Functional Analysis, New Age Internat. Publ., 1996.

[23] W. ŻElaZKo, Concerning topologization of $P(t)$, Acta Univ. Lodz. Folia Math., 8 (1996), 153-159.

[24] W. ŻELAZKo, On certain open problems in topological algebras, Rend. Sem. Mat. Fis. Milano, 59 (1989), 1992, 49-58.

[25] W. ŻELAZKo, On topologization of countably generated algebras, Studia Math., 112 (1994), 83-88.

[26] W. ŻELAZKo, Selected topics in topological algebra, Aarhus Univ. Lecture Notes 31, 1971. 